\title{
QUASIPARTICLE FORMATION AND DECAY IN PULSED PHOTOEXCITATION OF DISORDERED SEMICONDUCTORS
}

\author{
A. KaLvovía AND B. VelickÝ $\hat{Y}^{a, b}$ \\ ${ }^{a}$ Inst. of Phys., Czech Academy of Sciences \\ Na Slovance 2, 18040 Praha 8, Czech Republic \\ ${ }^{b}$ Faculty of Math. and Phys., Charles University \\ Ke Karlovu 5, 12116 Praha 2, Czech Republic
}

The quasiparticle states in strongly illuminated semiconductors are light hybridized electron and hole states, Galickii quasiparticles. Their properties, especially if they are photoexcited at small detunings, may be rather complex. A protracted formation period is followed by quantum beats of two decaying resonances corresponding to both sides of the hybridization gap. On an example of elastic scattering on an alloy disorder, these phenomena are demonstrated and analyzed in terms of poles of the retarded Green function and the corresponding residuals.

PACS numbers: 71.55.Eq, 72.10.Bg, 78.47.+p

\section{Introduction}

Quasiparticles, i.e., dressed electrons and holes, are used for decades as the basic notions in description of electric or optical transport processes in semiconductors. However, they are operationally relevant only in case that they act in the physical processes as a whole; it is thus required that a bare particle should convert to quasiparticle instantaneously, and further develop according to a simple evolution law resembling the bare particle evolution, perhaps with a renormalized dispersion law and with a finite life time. The excited state of the solid should be expressed in terms of the quasiparticle distribution. This latter question will be not treated in this paper, where we concentrate on the details of a single particle (electron or hole) excitation.

Usually the process of quasiparticle formation is very fast, but it can become anomalously long near scattering threshold or resonances. The relevant quantitative measure is the quasiparticle formation time in comparison with other characteristic times. Of these, the most important is the quasiparticle life time. These questions are best studied on behavior of an instantaneously injected one particle excitation, or, more precisely, of its retarded Green function (GF). For external 
disturbances strong enough, evolution of the quasiparticle exhibits coherent dependence on the external fields. We will address here the nonlinear optical excitation by a strong light pulse. Several extrinsically defined times enter the picture, like the pulse duration, and the Rabi period related to the transient optical hybridization of the conduction and valence one-electron states. For intense subpicosecond pulses, these times may become comparable with the duration of the quasiparticle formation and decay. The optical hybridization acts very anisotropically in the quasimomentum space, and the quasiparticle behavior is found to depend on the pulse characteristics in a manner correspondingly sensitive to the detuning of the bare photogenerated electron-hole pair. We will refer to the optically hybridized states of electrons and holes as to Galickii quasiparticles, and our aim is to study their short time transient behavior.

This problem of general importance becomes tractable in the case of purely elastic scattering, like by a static alloy disorder [1]. After configuration averaging, the evolution equations for the distribution become strictly decoupled from the one particle Dyson equation, and the latter can be solved using a generalized coherent potential approximation (CPA). All this will not be repeated here. We rather employ results obtained already for this particular case in order to develop a generalized definition of a quasiparticle state and analyze several characteristic situations.

\section{Galickii quasiparticles in a disordered semiconductor. Formal background}

We use a model semiconductor with two parabolic bands and direct gap, somewhat resembling GaAs. It is exposed to a strong stationary illumination with frequency $\omega$ and electric field amplitude $\mathcal{E}$, treated in the rotating wave approximation. Then, in the so-called Galickii representation, the action of illumination upon the electrons is given by a time-independent hybridization of both bands. The static disorder acts only in the conduction band, and it is described by a self-energy function $\sigma(z)$ which depends on the external field in the fully self-consistent CPA treatment and is defined in the closed upper half plane for the retarded GF, being analytic off the real axis [2]. The averaged Green function incorporating all these physical ingredients is diagonal with respect to the quasimomentum $k$, but not with respect to the band index. For one $k$, it is given, in the $c-v$ representation, as

$$
G^{r}(k, z)=\frac{1}{\mathcal{D}(z)}\left(\begin{array}{cc}
z-\epsilon_{v}-\hbar \omega & Q \\
Q & z-\epsilon_{c}-\sigma
\end{array}\right),
$$

where the $k$ and $z$ dependences are not indicated for convenience and

$$
\mathcal{D}(z)=\left(z-\epsilon_{c}-\sigma(z)\right)\left(z-\epsilon_{v}-\hbar \omega\right)-Q^{2} .
$$

Here, $\epsilon_{c}(k), \epsilon_{v}(k)$ are the bare bands, $\sigma(z)$ is a $k$-independent self-energy and the light induced hybridization $Q=e \mathcal{E}_{0} x_{c v}$ is taken as a real constant. In Fig. 1, we show, in the left hand panel, the bare bands, and the hybridization gap, as it would be created by the illumination in a crystal without disorder. The right hand panel shows the self-energy, which also develops a gap due to the illumination, but with 


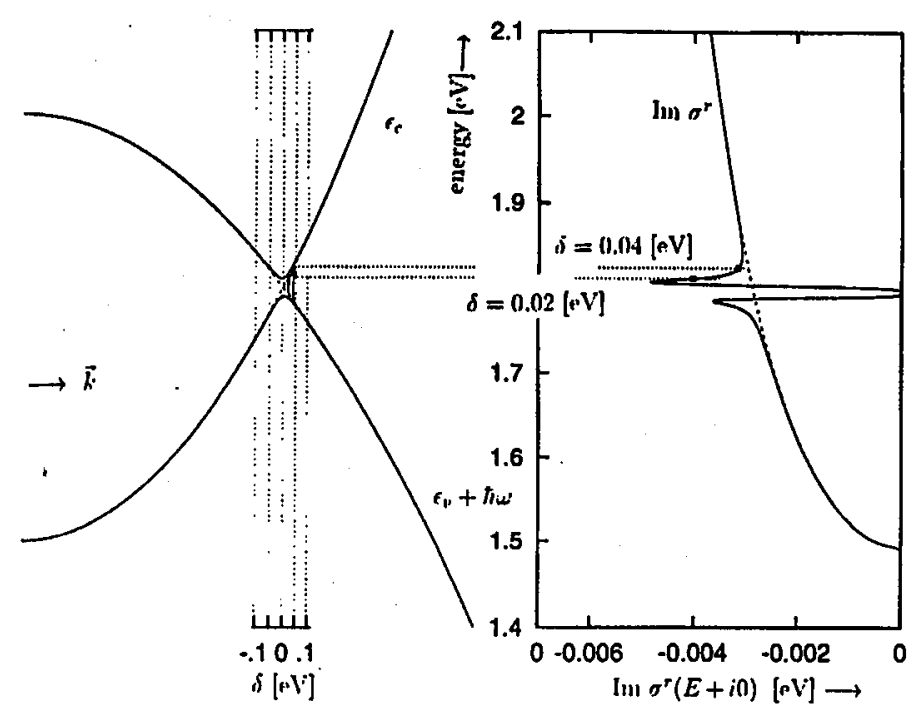

Fig. 1. Left: Bare band structure and bands hybridised by the light with the strength $Q=0.01 \mathrm{eV}$. Vertical dotted lines show detuning scale. Two arrows illustrate photogenerated $\mathrm{e}-\mathrm{h}$ pairs with detunings $\delta=0.02,0.04 \mathrm{eV}$. Right: Imaginary part of electron self-energy due to static disorder with optically induced gap. The dots, self-energy without illumination. The two points show the self-energies for detunings above.

a modified width [3]. In dots, the self-energy without illumination is shown for comparison. The time domain is attained by Fourier transform

$$
G^{\mathrm{r}}(k, t)=\int \frac{\mathrm{d} E}{2 \pi \hbar} G^{\mathrm{r}}(k, E+\mathrm{i} 0) \mathrm{e}^{-\mathrm{i} E t / \hbar} .
$$

Equations (1)-(3) form, once the self-energy is given, the full formal framework for our analysis.

\section{Definition and characteristics of the quasiparticles}

The "natural" way of finding quasiparticle energies and states is to find the poles (i.e., resonances) of the GF from the secular equation

$$
\mathcal{D}\left(z_{Q}\right)=0 \text {. }
$$

We will contrast two situations:

(A) $\sigma=\sigma^{\prime}+\mathrm{i} \sigma^{\prime \prime}=$ const, Wigner-Weisskopf model

(B) $\sigma$ is $z$-dependent, scattering included self-consistently; present paper.

In case (A), the $z$-dependence of the GF, or of related quantities is explicit. There are precisely two solutions of Eq. (4) with pole energies $z_{\ell}, z_{u}$. The Green function (1) can be exactly decomposed as

$$
\text { Case (A) } G(z)=\frac{P_{\ell}}{z-z_{\ell}}+\frac{P_{u}}{z-z_{u}},
$$

where the $k$-dependence is not indicated. The pole residues have the following properties of quasiparticle state projectors:

Case (A) $\quad P_{\ell}^{2}=P_{\ell}, \quad P_{u}^{2}=P_{u}, \quad P_{\ell} P_{u}=0, \quad P_{u}+P_{\ell}=1$. 
Finally, the corresponding time-dependent propagator is of the Wigner-Weisskopf form,

Case (A) $\quad G^{r}(t)=P_{\ell} \exp \left(-\mathrm{i} z_{\ell} t / \hbar\right)+P_{u} \exp \left(-\mathrm{i} z_{u} t / \hbar\right), t>0$.

In general, the effective Hamiltonian is non-Hermitian. Without hybridization, the electrons in the $c$-band would relax exponentially with the decay time $\hbar /\left|\sigma^{\prime \prime}\right|$. The hybridization transfers this damping also on the valence states. Both quasiparticle energies give rise to a finite quasiparticle life time. The quasiparticle projectors are non-Hermitian and, written as dyads, define the left and right quasiparticle states

Case (A) $\quad P_{u, \ell}=|u, \ell\rangle\langle\tilde{u}, \tilde{\ell}|, \quad P_{u, \ell}^{\dagger} \neq P_{u, \ell}$.

Only if $\sigma^{\prime \prime}=0$, the case of stable quasiparticles, the resonance energies are real, the survival amplitudes are not damped, and the quasiparticle projectors Hermitian. Case (A) thus appears as the general Wigner-Weisskopf quasiparticle model with finite life time.

In the case (B), when $\sigma$ becomes energy dependent, it may still be possible to find both poles from the "secular equation" (4) analytically continued to the non-physical sheet of its Riemann surface, and to construct operator residuals, e.g.,

$$
\begin{aligned}
& \text { Case (B) } R_{u}=\lim _{z \rightarrow z_{u}}\left(z-z_{u}\right) G(z)=(\mathrm{d} \mathcal{D}(z) / \mathrm{d} z)_{z=z_{u}}^{-1} \\
& \left(\begin{array}{cc}
z-\epsilon_{v}-\hbar \omega & Q \\
Q & z-\epsilon_{c}-\sigma\left(z_{u}\right)
\end{array}\right) .
\end{aligned}
$$

These residuals need not even be idempotent, but we may write the following spectral representation for $G(z)$ :

$$
\text { Case (B) } \quad G(z)=\mathcal{Z}_{u} \frac{P_{u}}{z-z_{u}}+\mathcal{Z}_{\ell} \frac{P_{\ell}}{z-z_{\ell}}+F(z) \equiv G_{\mathrm{P}}+F \text {. }
$$

As indicated, we separate the pole part $G_{\mathbf{P}}$ and the regular part $F$. Complex renormalization constants are introduced such that $P_{u, \ell}$ become idempotent. In contrast to (6), they do not form a decomposition of unity, because they belong to different resolvents

$$
\text { Case (B) } \quad P_{\ell}^{2}=P_{\ell}, \quad P_{u}^{2}=P_{u}, \quad P_{\ell} P_{u} \neq 0, \quad P_{u}+P_{\ell} \neq 1 .
$$

As a result, the two quasiparticle states interfere in the time domain

$$
\begin{gathered}
\text { Case (B) } G^{\mathrm{r}}(t)=\mathcal{Z}_{\ell} P_{\ell} \exp \left(-\mathrm{i} z_{\ell} t / \hbar\right)+\mathcal{Z}_{u} P_{u} \exp \left(-\mathrm{i} z_{u} t / \hbar\right)+F^{\mathrm{r}}(t) \\
\equiv G_{\mathbf{P}}^{\mathrm{r}}(t)+F^{\mathbf{r}}(t), \quad t>0 .
\end{gathered}
$$

It is expected that $F^{r}$ represents the quasiparticle formation and that it essentially vanishes after a quasiparticle formation time $\tau_{Q}$. The surviving exponentials of $G_{\mathrm{P}}^{\mathrm{r}}$ cannot be separated by any projection filtering because of the overlap (11). However, this mutual leakage of the quasiparticle states can be entirely absorbed in the initial condition for the Green function. To show this, we write the quasiprojectors $P_{u, \ell}$ in the form (8), as is always possible. We introduce biorthogonal vectors $|L\rangle,|U\rangle$, by

$$
\langle\ell \mid L\rangle=1,\langle\ell \mid U\rangle=0,\langle u \mid L\rangle=0,\langle u \mid U\rangle=1
$$



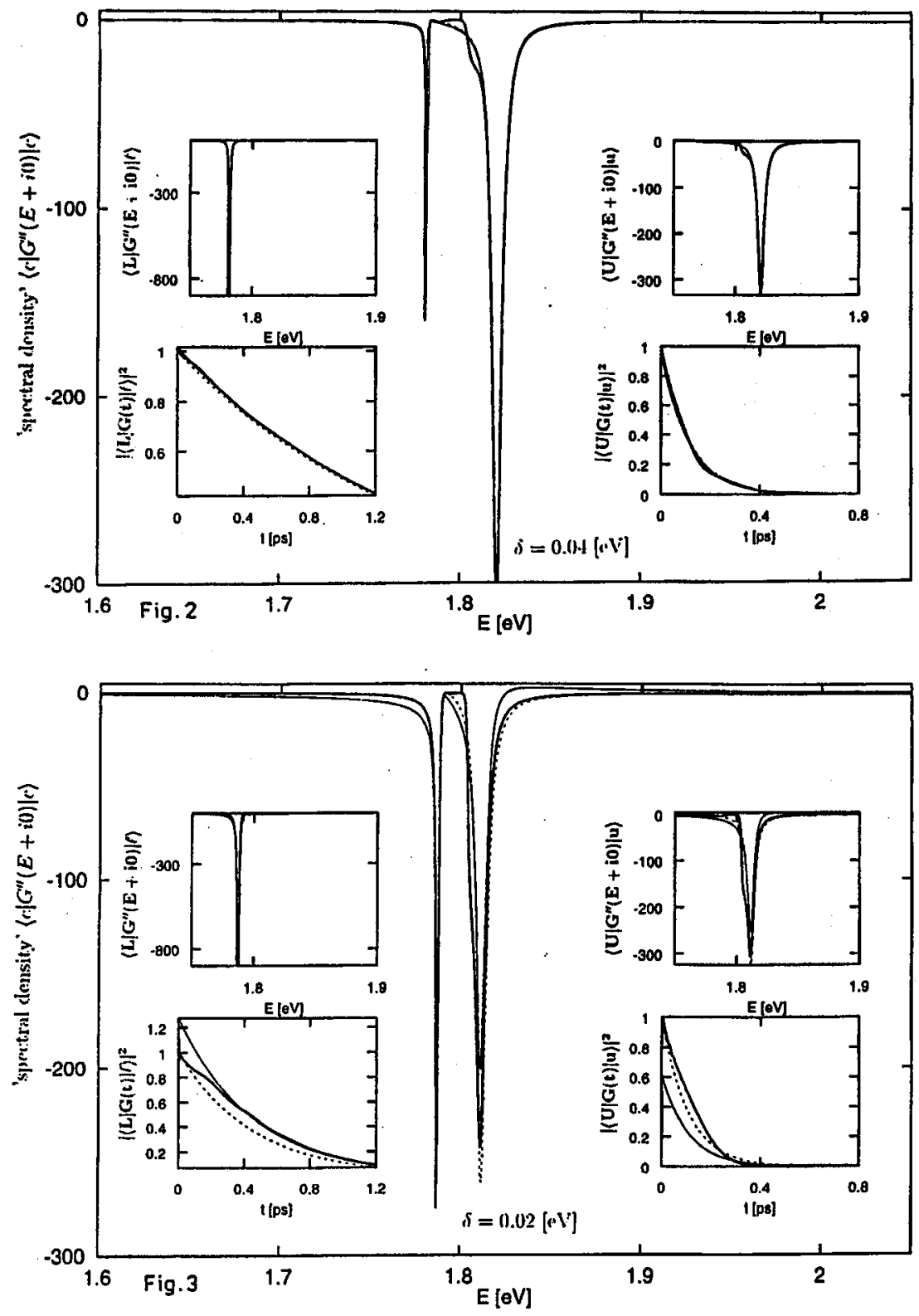

Fig. 2. cc-element of the anti-Hermitian part of GF as a function of energy for detuning $\delta=0.04$. Thick full line $-\left\langle c\left|G^{\mathrm{r}}(E+\mathrm{i} 0)\right| c\right\rangle$; thin full line $-\left\langle c\left|G_{\mathrm{P}}^{\mathrm{r}}(E+\mathrm{i} 0)\right| c\right\rangle$; dashed line $-\left\langle c\left|G_{\mathrm{W}}^{\mathrm{r}}(E+\mathrm{i} 0)\right| c\right\rangle$. The inserts show quasiparticle representation. Right hand panels: $\left\langle U\left|G^{\prime \prime}\right| u\right\rangle$ and $|\langle U|G(t)| u\rangle|^{2}$ for all three GF. Similar holds for $\ell$-elements on the left hand side.

Fig. 3. The same as in Fig. 2 but for detuning $\delta=0.02 \mathrm{eV}$. Notice the insufficiency of the quasiparticle picture, in contrast to the more regular case shown in Fig. 2. 
and define auxiliary projectors $\Pi_{u}$ and $\Pi_{\ell}$ having the usual properties (6):

$$
\Pi_{\ell}=|\ell\rangle\left\langle L\left|, \Pi_{u}=\right| u\right\rangle\langle U| \text {. }
$$

It is then easy to verify that

$$
\begin{aligned}
& G^{\mathrm{r}}(t)=G_{\mathrm{W}}^{\mathrm{r}}(t) Z+F^{\mathrm{r}}(t), \quad t>0 \\
& G_{\mathrm{W}}^{\mathrm{r}}(t)=\Pi_{\ell} \exp \left(-\mathrm{i} z_{\ell} t / \hbar\right)+\Pi_{u} \exp \left(-\mathrm{i} z_{u} t / \hbar\right), \\
& Z=\mathcal{Z}_{\ell} P_{\ell}+\mathcal{Z}_{u} P_{u}
\end{aligned}
$$

where $G_{\mathrm{W}}^{\mathrm{r}}$ may be termed the Wigner-Weisskopf propagalor and $Z$ is an operator renormalization constant.

\section{Results of numerical calculation}

While in the linear response the photoexcitation takes place strictly at the one-photon resonance (cross-over of the bands in Fig. 1), for a strong illumination, it spreads over the region of $k$-space, where the hybridization is noticeable. We present two cases for different detunings $\delta=\epsilon_{c}(k)-\epsilon_{v}(k)-\hbar \omega$ of the energy of the vertical pair in question. The detuning scale and both cases considered are shown in Fig. 1 , too. Both $\delta$ 's are positive, so that the $u$ state is $c$-like, the $l$ state is $v$-like. In Fig. 2, $\delta=0.04 \mathrm{eV}$. This falls already in the side wing of the hybridized area. The large plot shows the "spectral density" $\left\langle c\left|G^{\prime \prime}(E+\mathrm{i} 0)\right| c\right\rangle$, the $c c$ element of the anti-Hermitian part of the GF. The upper peak has a width $c a . \sigma^{\prime \prime}$, while the lower is rather sharp and its total relative strength is small. Along with the full $G^{\mathrm{r}}$, we consider also $G_{\mathrm{P}}^{\mathrm{r}}$ and $G_{\mathrm{W}}^{\mathrm{r}}$. They are shown in light and dashed lines, respectively. Only minor deviations are observed, which shows that the $F^{\mathbf{r}}$ contribution is small and that the renormalization effects are weak. The two peak structure would lead in the time domain to Rabi beats, which shows that the band representation is not suitable. The inserts relate to the quasiparticle representation. The right hand panels present the elements $\left\langle U\left|G^{\prime \prime}\right| u\right\rangle$ and $|\langle U|G(t)| u\rangle|^{2}$, again for all three GFs. They are not unrelated, for example,

$$
\begin{aligned}
& \left\langle U\left|G^{\mathrm{r}}(t)\right| u\right\rangle=\left\langle U\left|G_{\mathrm{P}}^{\mathrm{r}}(t)\right| u\right\rangle+\left\langle U\left|F^{\mathrm{r}}(t)\right| u\right\rangle \\
& =\left\langle U\left|G_{\mathrm{W}}^{\mathrm{r}}(t)\right| u\right\rangle \mathcal{Z}_{u}\langle\tilde{u} \mid u\rangle+\left\langle U\left|F^{\mathrm{r}}(t)\right| u\right\rangle .
\end{aligned}
$$

Similar holds for the $\ell$-elements on the left hand inserts. In Fig. 2, the filtering is good, the formation is fast and the $\ell$ quasiparticle decays rather slowly. For $\delta=0.02 \mathrm{eV}$ (Fig. 3), $G^{\mathbf{r}}$ and $G_{\mathbf{P}}^{\mathbf{r}}$ differ appreciably. The difference gives $F$. Indeed, pronounced formation effects are apparent until $t=0.4 \mathrm{ps}$, so that the $u$ particle dies before it got properly formed. Also the difference between $G_{\mathrm{P}}$ and $G_{\mathrm{W}}$ documents the effects of renormalization.

\section{Acknowledgment}

This work was supported by the Grant Agency of the Czech Republic under the project number 202/96/0098.

\section{References}

[1] P. Soven, Phys. Rev. 156, 809 (1967); B. Velický, S. Kirkpatrick, H. Ehrenreich, Phys. Rev. 175, 747 (1968).

[2] A. Kalvová, B. Velický, Z. Phys, B 94, 273 (1994); B. Velický, A. Kalvová, Phys. Status Solidi $B$ 188, 515 (1995).

[3] A. Kalvová, B. Velický, Z. Phys. B, in print; A. Kalvová, B. Velický, Acta Phys. Pol. A 90, 837 (1996). 